\title{
The effects of player grip on the dynamic behaviour of a tennis racket
}

\section{Delphine Chadefaux, Guillaume Rao, Jean-Loïc Le Carrou, Eric Berton \& Laurent Vigouroux}

To cite this article: Delphine Chadefaux, Guillaume Rao, Jean-Loïc Le Carrou, Eric Berton \& Laurent Vigouroux (2016): The effects of player grip on the dynamic behaviour of a tennis racket, Journal of Sports Sciences, DOI: 10.1080/02640414.2016.1213411

To link to this article: http://dx.doi.org/10.1080/02640414.2016.1213411

巴nublished online: 29 Jul 2016.

Submit your article to this journal $\sqsubset \pi$

Џ Article views: 26

View related articles 5

View Crossmark data ¿ 


\title{
The effects of player grip on the dynamic behaviour of a tennis racket
}

\author{
Delphine Chadefauxa, Guillaume Rao ${ }^{a}$, Jean-Loïc Le Carrou ${ }^{b}$, Eric Berton ${ }^{\mathrm{a}}$ and Laurent Vigouroux ${ }^{\mathrm{a}}$ \\ ${ }^{a}$ Aix Marseille Univ, CNRS, ISM, Inst Movement Sci, Marseille, France; 'borbonne Universités, UPMC Univ Paris 06, CNRS, UMR 7190, LAM - Institut
} Jean le Rond d'Alembert, Paris, France

\begin{abstract}
The aim of this article is to characterise the extent to which the dynamic behaviour of a tennis racket is dependent on its mechanical characteristics and the modulation of the player's grip force. This problem is addressed through steps involving both experiment and modelling. The first step was a free boundary condition modal analysis on five commercial rackets. Operational modal analyses were carried out under "slight", "medium" and "strong" grip force conditions. Modal frequencies and damping factors were then obtained using a high-resolution method. Results indicated that the dynamic behaviour of a racket is not only determined by its mechanical characteristics, but is also highly dependent on the player's grip force. Depending on the grip force intensity, the first two bending modes and the first torsional mode frequencies respectively decreased and increased while damping factors increased. The second step considered the design of a phenomenological hand-gripped racket model. This model is fruitful in that it easily predicts the potential variations in a racket's dynamic behaviour according to the player's grip force. These results provide a new perspective on the player/ racket interaction optimisation by revealing how grip force can drive racket dynamic behaviour, and hence underlining the necessity of taking the player into account in the racket design process.
\end{abstract}

\section{ARTICLE HISTORY}

Accepted 5 July 2016

\section{KEYWORDS}

Grip force; vibration; tennis racket; phenomenological model; hand-tools interaction

\section{Introduction}

The interaction between the tennis player and his/her racket is crucial for performance, prevention of pathologies and comfort (Brody, 1979; Cross, 2000; Hennig, 2007). Consequently, over the past few decades, a wide range of racket mechanical parameters has been investigated and improved (e.g., geometry, materials, mass distribution, stringing) to optimise this player/racket interaction (Miller, 2006).

From the player's point of view, stroke-induced vibration is considered a key parameter for comfort (Stroede, Noble, \& Walker, 1999) and in preventing the condition of tennis elbow (Brody, 1989; Hatze, 1976; Hennig, 2007; Wei, Chiang, Shiang, \& Chang, 2006) since the induced vibration is directly transmitted to the body. Research has thus been concentrated on the amount of vibration delivered to the player, leading to the investigation of the racket's first vibration node, i.e., the impact position where the vibration energy transmitted to the forearm is minimal (Brody, 1979; Cross, 2000). Nevertheless, no straightforward relationship has been demonstrated between strokeinduced vibration and the player's feeling. The most likely reason is the complexity of the player/racket interaction, as it has already been pointed out that players are able to fine-tune their grip force according to the stroke performance (Savage \& Subic, 2006), and to the racket properties for a given identical stroke (Rossi, Vigouroux, Barla, \& Berton, 2014). However, the player/ racket interaction is not fully understood, hindering the design of rackets well-adapted to the players.
Knowing that the way the racket is held affects its dynamic behaviour, several investigations have focused on the comparison between hand-gripped, freely suspended and clamped tennis rackets. As expected, it was observed that the vibrational waves in a tennis racket were more rapidly damped when held than when freely suspended (Brody, 1987, 1989; Hatze, 1976; Hennig, Rosenbaum, \& Milani, 1992). Interestingly, it has also been shown that the tighter the grip force, the faster the vibrations will be dampen (Savage, 2006), and that holding a racket induces modal frequency shifts toward lower frequencies with respect to the freely suspended boundary condition (Banwell, Roberts, Halkon, Rothberg, \& Mohr, 2014). Although grip force is expected to be highly player-dependent, no investigation has demonstrated how grip force modulation would affect the dynamic behaviour of the racket as a function of its mechanical characteristics. This article proposes an experiment-based analysis of racket dynamic behaviour as a function of the applied grip force and then introduces a phenomenological model of a handgripped racket.

\section{Methods}

\subsection{Experimental modal analysis of rackets under free boundary conditions}

The experimental modal analysis of five commercial rackets, whose mechanical characteristics are given in Table 1, was 
Table 1. Mechanical characteristics of the rackets involved in the study.

\begin{tabular}{|c|c|c|c|c|c|}
\hline & Wilson & Prince & Artengo & Babolat & Head \\
\hline & Triad 6 & $\mathrm{O} 3$ & TR820 & $\begin{array}{c}\text { AeroPro } \\
\text { Drive }\end{array}$ & \multirow{2}{*}{$\frac{\text { Prestige }}{\left(R_{5}\right)}$} \\
\hline & $\left(R_{1}\right)$ & $\left(\mathrm{R}_{2}\right)$ & $\left(R_{3}\right)$ & $\left(\mathrm{R}_{4}\right)$ & \\
\hline Head surface $\left(\mathrm{cm}^{2}\right)$ & 742 & 742 & 645 & 645 & 600 \\
\hline Mass $(\mathrm{g})$ & 253 & 259 & 282 & 304 & 327 \\
\hline Length $(\mathrm{cm})$ & 69.8 & 69.0 & 68.7 & 68.7 & 68.5 \\
\hline Moment of inertia $\left(\mathrm{kg} \cdot \mathrm{m}^{2}\right)$ & 0.0311 & 0.0300 & 0.0266 & 0.0299 & 0.0297 \\
\hline Strings brand & \multicolumn{5}{|c|}{ Artengo TA Feel } \\
\hline Strings tension (kg) & \multicolumn{5}{|c|}{$21 / 20$} \\
\hline
\end{tabular}

Note that the moments of inertia were measured about the axis perpendicular to the plane of the racket through a pivot point $10 \mathrm{~cm}$ from the butt of the handle and that classical orders of magnitude have been obtained.

carried out by extracting mode shapes, eigenfrequencies and damping factors from frequency response functions (FRFs) directly measured on each racket under free boundary conditions (i.e., rackets supported on elastic cords). These boundary conditions were chosen since they have been shown to be the best representation of the dynamic behaviour of a hand-held racket among the classical boundary conditions (Cross, 1997; Kotze, Mitchell, \& Rothberg, 2000). FRFs are then defined by

$$
H_{i}(\omega)=\frac{A(\omega)}{F_{i}(\omega)},
$$

where $\omega=2 \pi f$ is the angular frequency, $H_{i}$ refers to the FRFs on a 22-point mesh distributed over the racket structure (see Figure 1), $F_{i}$ is the excitation force at the ith point on the mesh provided by an impulse hammer (PCB Piezotronics; $\pm 400 \mathrm{~N}$ pk; resonant frequency $\geq 15 \mathrm{kHz}$ ) and $A$ is the resulting normal acceleration measured by a single-axis accelerometer (PCB Piezotronics; $\pm 500 \mathrm{~g}$ pk; $[2-10,000] \mathrm{Hz}$, sampling rate set at $4000 \mathrm{~Hz}$ ) bonded onto the left shoulder of the racket. For each of the 22 FRFs, the excitation force and the acceleration signals were checked both in the temporal and the frequency domains. As many measures as necessary were reproduced until the signals were considered acceptable (i.e., no secondary hammer impact, satisfactory bandwidth and noise level based on the coherence function). An uniform rectangular and an exponential decay windows were applied on the excitation force and the acceleration signals (note that the artificial damping introduced by windowing the normal acceleration signals is $10^{5}$ times lower than the actual damping of the rackets, and therefore negligible). These 22 measurements are made up to $1000 \mathrm{~Hz}$ on account of the bandwidth limit of the impulse hammer. Finally, modal identification was carried out using the least-squares complex frequency-domain (LSCF) method (Guillaume, Verboven, Vanlanduit, Van Der Auweraer, \& Peeters, 2003; Piranda, 2001) implemented in the Modan software developed at the FEMTO-ST institute (http://modan.dma-softs.com/).

\subsection{Operational modal analysis of hand-gripped rackets}

The dynamic behaviour of the same five rackets were analysed in the hand-gripped state. A single female participant (28 years old, $163 \mathrm{~cm}$ tall, $58 \mathrm{~kg}$, right hand length $17.5 \mathrm{~cm}$ ) was involved in the study. The experiment was approved by the local ethic committee and the participant signed a consent form. She was asked to hold each racket handle with the same hand positioning corresponding to a forehand eastern grip. The consistency of the hand positioning was verified according to the pressure repartition on pressure maps wrapped around the racket's handles (Hoof Sensor 3200, Tekscan, Boston, USA). The map was $558.8 \mathrm{~mm} \times 181.6 \mathrm{~mm}$ and

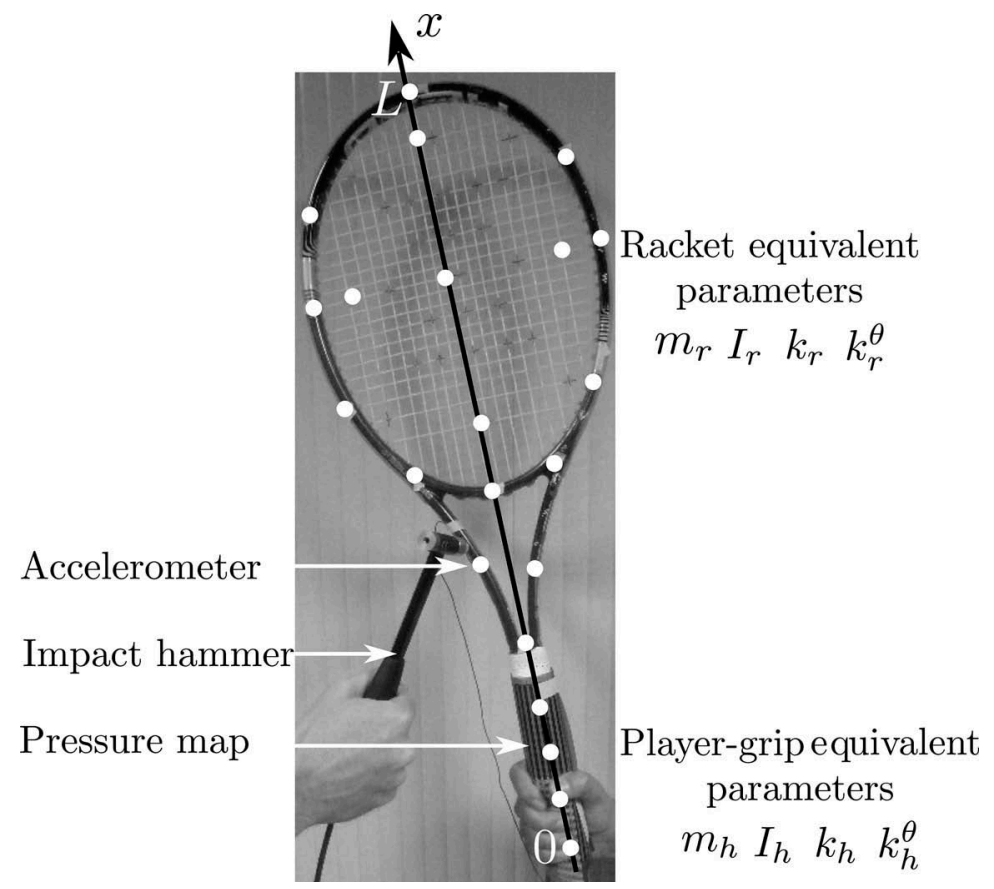

Figure 1. Investigation of the dynamic behaviour of a hand-gripped racket. Picture of the experimental set-up with the equivalent parameters involved in the handgripped racket modelling. White circles highlight the 22-point mesh used for the classical modal analysis under free boundary conditions. 
$0.228 \mathrm{~mm}$ thick, consisted into a $33 \times 33$ sensors matrix, had a spatial resolution of 3.9 sensors per $\mathrm{cm}^{2}$, and a dynamic range of $2068 \mathrm{kPa}$. The sampling rate was set at $F_{\mathrm{s}}=50 \mathrm{~Hz}$. Three different grip force intensities were successively investigated, referred to as "slight", "medium" and "strong". These grip force intensities were meant to investigate the dynamic behaviour of tennis rackets under a wide range of grip forces, from the hand presence on the handle ("slight") to actual grip force intensities during tennis strokes ("medium" and "strong"). Five measurements per condition were collected. The applied grip forces were controlled by measuring the total amount of pressure applied at each instant on the pressure maps. To gain insight into the corresponding grip force, the pressure maps were first calibrated using weights over a range of $600 \mathrm{~N}$ corresponding to the normal range of grip forces (Rossi, Berton, Grélot, Barla, \& Vigouroux, 2012). However, although these sensors were good enough to compare different conditions, they were not accurate enough to precisely estimate the force magnitude (Rossi et al., 2014). A grip index was therefore determined for each trial by normalising the estimated grip forces by the maximum grip force value estimated on the related pressure map. Considering the practical difficulty experienced by the participant in keeping a constant grip force in a static position for the purposes of a 22-point modal analysis, only one colocalised FRF was measured for each racket at its left shoulder (see Figure 1). The excitation force was provided using the same impulse hammer as before, and the resulting normal acceleration was also measured by the same single-axis accelerometer. In order to extract modal parameters from these single measurements, we assumed the gathered signals could be described by the noiseless exponential sinusoidal model (ESM)

$$
s(t)=\sum_{n=1}^{N} a_{n} \cos \left(2 \pi f_{n} t+\phi_{n}\right) e^{-2 \pi f_{n} \delta_{n} t},
$$

where the index $n$ indicates the components required to properly describe the signal, $N$ is the model order, $t$ is the dimensionless time vector, $a_{n}$ the positive amplitude of the $n$th component, $f_{n} \in\left[-\frac{1}{2} ; \frac{1}{2}\right]$ is the frequency, $\phi_{k} \in[-\pi ; \pi]$ the phase and $\delta_{n}$ the damping factor. A high-resolution method (ESPRIT: estimation of signal parameters via rotational invariance techniques) was then used to accurately estimate the modal parameters $\left(a_{n}, f_{n}, \phi_{n}\right.$ and $\left.\delta_{n}\right)$ in each recorded signal (Le Carrou, Gautier, \& Badeau, 2009; Paté, Le Carrou, \& Fabre, 2014; Roy, Paulraj, \& Kailath, 1986).

\subsection{Hand-gripped racket modelling}

Based on the Rayleigh-Ritz method, a model of the handgripped racket was developed to predict how the player's grip force affects the dynamic behaviour of the racket. According to Cross and Nathan (2009), despite its non-uniform shape, a tennis racket has an approximately uniform mass distribution along its central axis, and behaves similarly to a uniform beam. Therefore, the racket was modelled as a uniform beam of equivalent mass $m_{r}=\rho S L$, equivalent moment of inertia about its central axis through its centre of mass
$I_{\mathrm{r}}=\rho I_{0} L$, equivalent stiffness $k_{\mathrm{r}}=\frac{E l_{0}}{L^{3}}$ and equivalent torsional stiffness $k_{\mathrm{r}}^{\theta}=\frac{\mu l_{0}}{L}$, where $\rho, L, S, I_{0}$ and $E$ are the beam's volumetric mass density, length, cross-sectional area, area moment of inertia and Young's modulus, respectively.

The grip force model comprised linear and torsional springs of stiffnesses $k_{h}$ and $k_{h}^{\theta}$ connected to a cylindrically shaped mass $m_{h}$ of moment of inertia $I_{h}$ and radius $r_{h}$ rigidly fixed to one end of the racket. Theoretical developments, details of which are presented in Appendix, lead to the classical equation

$$
\left|\mathbb{K}-\mathbb{M} \omega^{2}\right|=\{0\}
$$

$\mathbb{K}$ and $\mathbb{M}$ are square matrices containing the bending and torsional stiffnesses and masses of the system, namely

$$
\mathbb{K}=\left[\begin{array}{cc}
\mathbb{K}_{1} & \mathbb{O}_{N_{\mathrm{b}}, N_{\mathrm{t}}} \\
\mathbb{O}_{N_{\mathrm{t}}, N_{\mathrm{b}}} & \mathbb{K}_{2}
\end{array}\right]
$$

and

$$
\mathbb{M}=\left[\begin{array}{cc}
\frac{m_{\mathrm{r}}}{2} \mathbb{I}_{N_{\mathrm{b}}}+m_{\mathrm{h}}(-1)^{i+j} & \mathbb{O}_{N_{\mathrm{b}}, N_{\mathrm{t}}} \\
\mathbb{O}_{N_{\mathrm{t}}, N_{\mathrm{b}}} & \frac{l_{\mathrm{r}}}{2} \mathbb{I}_{N_{\mathrm{t}}}+I_{\mathrm{h}}(-1)^{i+j}
\end{array}\right],
$$

where $\mathbb{K}_{1}=\operatorname{diag}\left\{\frac{8 \pi^{4}}{2} k_{\mathrm{r}}, \ldots, \frac{\left(N_{\mathrm{b}}+1\right)^{4} \pi^{4}}{2} k_{\mathrm{r}}\right\}+k_{\mathrm{h}}(-1)^{i+j}$,

$\mathbb{K}_{2}=\operatorname{diag}\left\{\frac{4 \pi^{2}}{2} k_{\mathrm{r}}^{\theta}, \ldots, \frac{\left(N_{\mathrm{t}}+1\right)^{2} \pi^{2}}{2} k_{\mathrm{r}}\right\}+k_{\mathrm{h}}^{\theta}(-1)^{i+j}, i$ and $j$ are the indices for the matrices' rows and columns, and $N_{\mathrm{b}}$ and $N_{\mathrm{t}}$ are the numbers of degrees of freedom in bending and torsion, respectively. $N_{\mathrm{b}}$ and $N_{\mathrm{t}}$ were determined based on the experimental investigation of how grip force modulation affect the dynamic behaviour of the racket, conveying finally to $N_{\mathrm{b}}=2$ and $N_{\mathrm{t}}=1$.

The beam's equivalent mass $m_{r}$ is taken to be the same as the racket's effective mass, as given in Table 1, and the first solution step consists in extracting the beam's equivalent parameters $k_{\mathrm{r}}, l_{\mathrm{r}}$ and $k_{\mathrm{r}}^{\theta}$. These can be estimated under the free boundary condition (i.e., $m_{\mathrm{h}}=k_{\mathrm{h}}=I_{\mathrm{h}}=k_{\mathrm{h}}^{\theta}=0$ ) by minimising Equation (3) where $\{\omega\}$ were experimentally extracted from a preliminary modal analysis (see Section 2.1).

\subsection{Applications of the model}

The hand-gripped racket model can be used for two purposes. First, the model can be inverted to determine the set of equivalent player-grip parameters $\left\{k_{\mathrm{h}}, l_{\mathrm{h}}, k_{\mathrm{h}}^{\theta}\right\}$ based on the measured modal frequencies, while $m_{\mathrm{h}}$ is set to an estimate of the player's actual hand mass based on anthropometric tables (Zatsiorsky, 2002). This process is applied in this study to estimate typical orders of magnitude for these parameters depending on the various grip force intensities. Second, the model is able to determine the modal frequencies of a handgripped racket for a given set of player-grip equivalent parameters $\left\{m_{\mathrm{h}}, k_{\mathrm{h}}, l_{\mathrm{h}}, k_{\mathrm{h}}^{\theta}\right\}$. To this end, $k_{\mathrm{h}}, l_{\mathrm{h}}$ and $k_{\mathrm{h}}^{\theta}$ were considered over the range of values initially obtained from the model under different grip force intensities while $m_{\mathrm{h}}$ is considered up to $0.6 \mathrm{~kg}$ (Zatsiorsky, 2002). The computed modal frequencies are subsequently denoted $\tilde{f}$. 


\subsection{Statistical analysis}

For each racket, mean values of each estimated modal frequency and damping factor were calculated over the five trials collected for each grip force condition. Spearman rank correlations were computed to identify the relationships between grip force intensity and the racket's dynamic behaviour while Pearson correlations were computed to identify the relationships between the rackets' mechanical properties and their dynamic behaviour. They are subsequently referred to as $R_{s}$ and $R_{p}$, respectively. For all statistical comparisons, significance levels were set at $p=0.05$.

\section{Results}

\subsection{Modal behaviour of the rackets under free boundary conditions}

The results of the modal analyses under free boundary conditions are illustrated in Figure 2(a) by a typical FRF magnitude curve and presented in Table 2 . Three bending modes $\left(B_{1}, B_{2}\right.$, $\left.B_{3}\right)$, two torsional modes $\left(T_{1}, T_{2}\right)$, as well as three string-bed modes $\left(\mathrm{S}_{1}, \mathrm{~S}_{2}, \mathrm{~S}_{3}\right)$ are highlighted. All the string-bed modes gave a vibrational node where the hand is expected to be on the racket handle. Additionally, the second torsional mode as well as the first two bending modes gave vibrational antinodes at this same position. The frequencies and damping factors of these highlighted modes are denoted subsequently by $f_{\mathrm{B}_{1,2,3} / \mathrm{T}_{1,2} / \mathrm{S}_{1,2,3}}$ and $\delta_{\mathrm{B}_{1,2,3} / \mathrm{T}_{1,2} / \mathrm{S}_{1,2,3}}$.

\subsection{Change in the modal behaviour of the racket with grip force}

For each of the 25 investigated conditions (5 rackets, 5 trials), the total grip force applied during measurement of the FRF was estimated. "Slight", "medium" and "strong" grip force intensities correspond to averaged factors of $4 \pm 1 \%, 25 \pm$ $6 \%$ and $78 \pm 8 \%$ of the maximum grip force value achieved by the participant during the session, respectively. All standard deviations computed over the measured FRFs were less than $1 \mathrm{~dB}$ and each averaged about $0.1 \mathrm{~dB}$ per condition over the frequency range (Figure 2(b)). FRFs are affected by the grip force intensity: while shifts in modal frequencies occurred, the damping factors greatly increased with grip force intensity. For each racket investigated, $f_{\mathrm{B}_{1}}$ and $f_{\mathrm{B}_{2}}$ decreased when the grip force increased $\left(\mathrm{R}_{\mathrm{s}}=-0.65, p=0.01\right.$ and $\left.\mathrm{R}_{\mathrm{s}}=-0.77, p<0.01\right)$. When increasing the grip force from "slight" to "strong" (+ 74 $\pm 8 \%$ ), both $f_{\mathrm{B}_{1}}$ and $f_{\mathrm{B}_{2}}$ decreased on average by $15 \pm 5 \mathrm{~Hz}$ $(-10 \pm 1 \%)$ and $16 \pm 4 \mathrm{~Hz}(-4.1 \pm 0.6 \%)$, respectively (Figure 3 ). On the other hand, $f_{\mathrm{T}_{1}}$ increased with grip force $\left(R_{s}=0.83, p<0.01\right)$, giving a $6 \pm 1 \mathrm{~Hz}$ increase $(+1.5 \pm$ $0.3 \%)$.

The results indicated that damping factors were greatly increased by the presence of a hand. For instance, in the "medium" condition, $\delta_{\mathrm{B}_{1}}, \delta_{\mathrm{B}_{2}}$ and $\delta_{\mathrm{T}_{1}}$ were estimated to be $156 \pm 45 \%, 1005 \pm 481 \%$, and $312 \pm 159 \%$ of their values under free boundary conditions. When increasing the grip force from "slight" to "strong", damping factors $\delta_{\mathrm{B}_{1}}, \delta_{\mathrm{B}_{2}}$, and

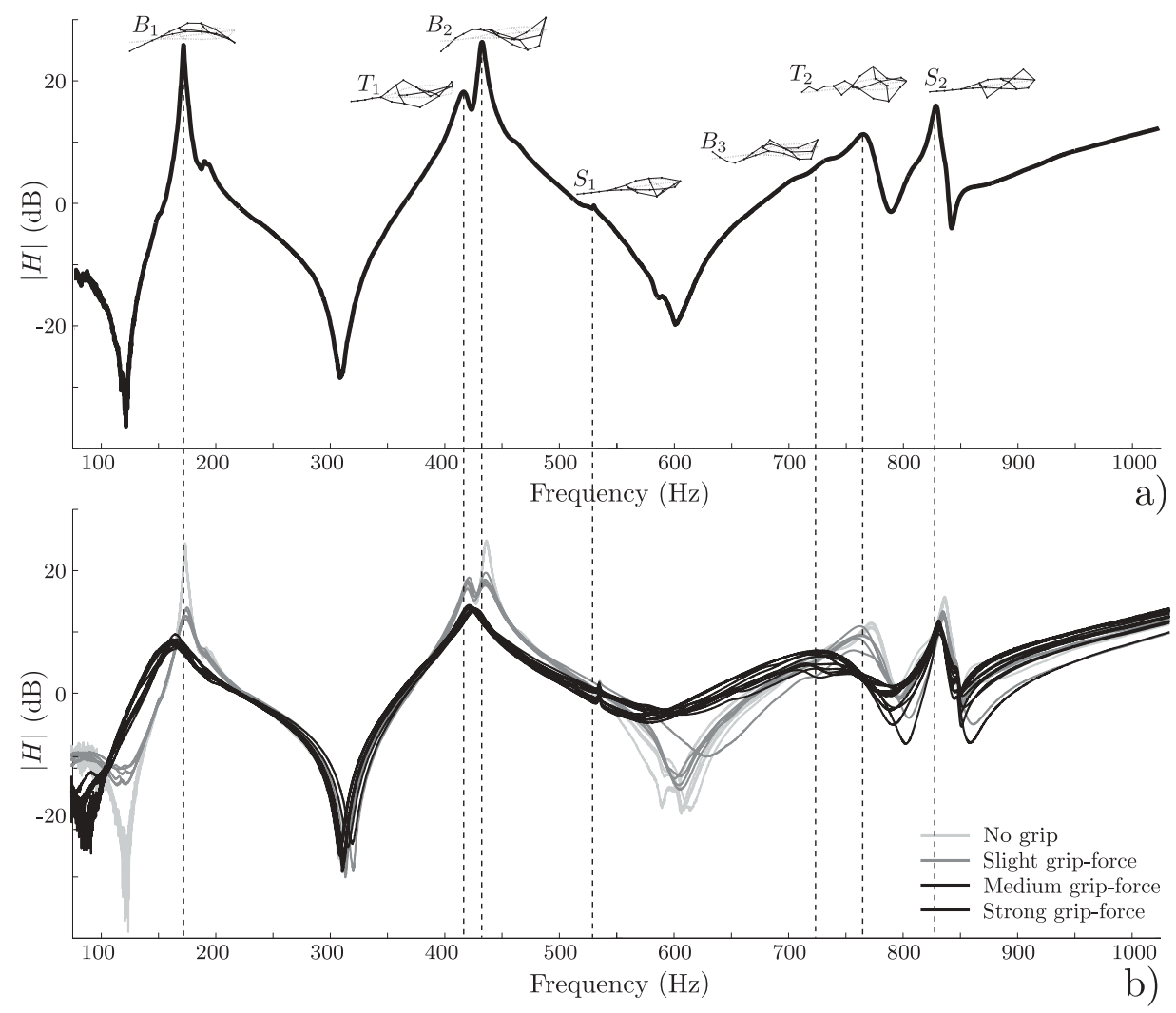

Figure 2. Colocalised frequency response function measured on the racket $R_{5}$ at its left shoulder. (a) Racket under free boundary conditions. Bending modes ( $B_{n}$ ), torsional modes $\left(T_{n}\right)$, as well as string-bed modes $\left(S_{n}\right)$ are highlighted. Mode shapes are given at their corresponding modal frequencies. Being higher than $1000 \mathrm{~Hz}$ the third string-bed mode frequency does not appear in the figure. (b) Racket under free boundary conditions and hand-held at three grip force conditions. Five measurements are provided for each grip force intensity. 
Table 2. Tennis rackets' modal shapes, frequencies $(f)$ and damping factors $(\delta)$ obtained by classical modal analysis.

\begin{tabular}{|c|c|c|c|c|c|c|c|c|}
\hline Modes & & & $\mathrm{R}_{1}$ & $\mathrm{R}_{2}$ & $\mathrm{R}_{3}$ & $\mathrm{R}_{4}$ & $\mathrm{R}_{5}$ & Modal shapes \\
\hline \multirow[t]{6}{*}{ Bending } & \multirow[t]{2}{*}{$\mathrm{B}_{1}$} & $f(\mathrm{~Hz})$ & 145.9 & 151.8 & 140.0 & 150.5 & 138.6 & \\
\hline & & $\delta(\%)$ & (1.4) & (0.5) & (0.6) & (0.7) & (0.6) & \\
\hline & \multirow[t]{2}{*}{$\mathrm{B}_{2}$} & $f(\mathrm{~Hz})$ & 436.6 & 357.8 & 387.2 & 415.9 & 381.7 & \\
\hline & & $\delta(\%)$ & (1.7) & $(0.6)$ & $(0.9)$ & $(0.7)$ & $(0.5)$ & \\
\hline & \multirow[t]{2}{*}{$\mathrm{B}_{3}$} & $f(\mathrm{~Hz})$ & 824.8 & 676.3 & 740.9 & 755.6 & 707.4 & \\
\hline & & $\delta(\%)$ & (1.7) & $(0.7)$ & $(0.8)$ & $(0.8)$ & $(0.8)$ & \\
\hline \multirow[t]{4}{*}{ Torsion } & \multirow[t]{2}{*}{$\mathrm{T}_{1}$} & $f(\mathrm{~Hz})$ & 357.6 & 367.1 & 366.0 & 395.9 & 366.1 & \\
\hline & & $\delta(\%)$ & (1.4) & (0.6) & (1.2) & (0.7) & (1.4) & \\
\hline & \multirow[t]{2}{*}{$\mathrm{T}_{2}$} & $f(\mathrm{~Hz})$ & 951.9 & 1070.0 & 886.5 & 953.5 & 876.6 & \\
\hline & & $\delta(\%)$ & (1.6) & $(0.4)$ & (0.9) & $(0.7)$ & (0.7) & \\
\hline \multirow[t]{6}{*}{ String-bed } & \multirow[t]{2}{*}{$\mathrm{S}_{1}$} & $f(\mathrm{~Hz})$ & 501.9 & 477.6 & 511.3 & 505.0 & 515.3 & \\
\hline & & $\delta(\%)$ & (0.8) & $(0.1)$ & (0.2) & $(0.2)$ & $(0.2)$ & \\
\hline & \multirow[t]{2}{*}{$\mathrm{S}_{2}$} & $f(\mathrm{~Hz})$ & 760.1 & 754.2 & 765.9 & 806.1 & 818.7 & \\
\hline & & $\delta(\%)$ & (0.3) & $(0.2)$ & $(0.2)$ & $(0.3)$ & $(0.2)$ & \\
\hline & \multirow[t]{2}{*}{$\mathrm{S}_{3}$} & $f(\mathrm{~Hz})$ & 795.5 & 774.1 & 814.5 & 1089.3 & 1079.8 & \\
\hline & & $\delta(\%)$ & $(0.2)$ & $(0.2)$ & $(0.2)$ & $(0.2)$ & $(0.1)$ & \\
\hline
\end{tabular}

$\mathrm{R}_{1 \ldots 5}$ referred to rackets Wilson Triad 6, Prince 03, Artengo TR820, Babolat AeroPro Drive and Head Prestige.

$\delta_{\mathrm{T}_{1}}$ increased on an average by $0.2 \pm 0.5 \%, 4.0 \pm 2.1 \%$ and $0.9 \pm 0.2 \%$, respectively (Figure 3 ). For each racket investigated, $\delta_{B_{2}}$ and $\delta_{T_{1}}$ increased when the grip force increased $\left(R_{s}\right.$ $=0.76, p<0.01$ and $R_{S}=0.60, p=0.02$ ) while no significant effect existed for $\delta_{\mathrm{B}_{1}}\left(\mathrm{R}_{\mathrm{s}}=0.36, p=0.18\right)$.

For all the rackets investigated, as expected, the first stringbed mode was not affected by the grip force intensity. For modes above $600 \mathrm{~Hz}$, no straightforward common behaviour was observed: each racket response differs from that of the others.

Investigation of the relationships between the racket properties as given in Table 1 and the evolution of their dynamic behaviour showed that the higher the frame stiffness, the more slowly $f_{B_{1}}$ decreased with grip force $\left(R_{p}=0.93\right.$, $p=0.02$ ). Moreover, the decrease in $f_{B_{2}}$ with grip force is related to the moment of inertia of the racket. A low moment of inertia resulted in a drop between the "slight" and the "medium" conditions $\left(R_{p}=-0.92, p=0.03\right)$ which is slower than between the "medium" and the "strong" conditions $\left(\mathrm{R}_{\mathrm{p}}=\right.$ $-0.91, p=0.03$ ). Finally, under "medium" and "strong" conditions, the results indicate that $\delta_{\mathrm{T}_{1}}$ decreased with racket length $\left(R_{p}=-0.89, p=0.04\right.$ and $\left.R_{p}=-0.92, p=0.03\right)$ while it increased with frame stiffness $\left(R_{p}=0.97, p<0.01\right.$ and $R_{p}=$ $0.88, p=0.049$ ).

\subsection{Estimating the racket equivalent parameters}

As per the previous results, the dynamic behaviour of the handgripped rackets was investigated throughout the modes $B_{1}, B_{2}$ and $T_{1}$ using the phenomenological model (Equation (3)), i.e., $N_{\mathrm{b}}=2$ and $N_{\mathrm{t}}=1$. The equivalent beam parameters $\left\{k_{\mathrm{r}}, l_{\mathrm{r}}, k_{\mathrm{r}}^{\theta}\right\}$ estimated for the five rackets are shown in Table 3: $k_{\mathrm{r}}=211.1 \pm 28.4 \mathrm{~N} \cdot \mathrm{m}^{-1}, \quad I_{\mathrm{r}}=0.0218 \pm 0.0002 \mathrm{~kg} \cdot \mathrm{m}^{2}$ and

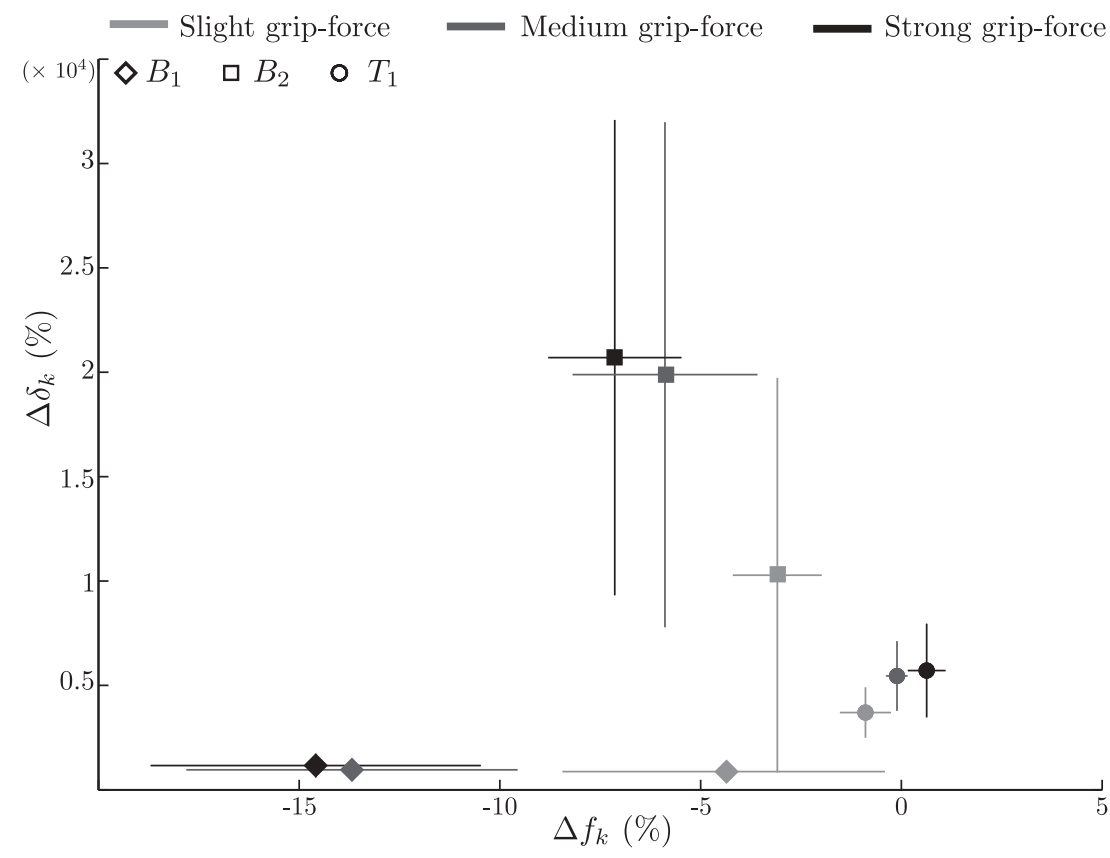

Figure 3. Measured evolution of normalised modal frequencies $\left(f_{\mathrm{B} 1}, f_{\mathrm{B} 2}, f_{\mathrm{T} 1}\right)$ and damping factors $\left(\delta_{\mathrm{B}_{1}}, \delta_{\mathrm{B}_{2}}, \delta_{\mathrm{T}_{1}}\right)$ according to grip force intensity, where indices $\mathrm{B} 1$, $\mathrm{B} 2$ and T1 referred to the two first bending modes and the first torsional mode, respectively. $\Delta f_{n}=\frac{f_{n}-f_{n}^{0}}{f_{n}^{0}}$ and $\Delta \delta_{n}=\frac{\delta_{n}-\delta_{n}^{0}}{\delta_{n}^{0}}$ where the reference frequencies $\left(f_{\mathrm{B} 1}^{0}, f_{\mathrm{B} 2}^{0}, f_{\mathrm{T} 1}^{0}\right)$ and damping factors $\left(\delta_{\mathrm{B}_{1}}^{0}, \delta_{\mathrm{B}_{2}}^{0}, \delta_{\mathrm{T}_{1}}^{0}\right)$ are measured under free boundary conditions. 
Table 3. Beam equivalent parameters estimated for each racket investigated based on the two first bending modes and the first torsional mode frequencies.

\begin{tabular}{lccccc}
\hline & $\mathrm{R}_{1}$ & $\mathrm{R}_{2}$ & $\mathrm{R}_{3}$ & $\mathrm{R}_{4}$ & $\mathrm{R}_{5}$ \\
\hline$k_{\mathrm{r}}\left(\mathrm{N} \cdot \mathrm{m}^{-1}\right)$ & 222.0 & 163.4 & 198.7 & 247.2 & 224.2 \\
$l_{\mathrm{r}}\left(\mathrm{kg} \cdot \mathrm{m}^{2}\right)$ & 0.0220 & 0.0218 & 0.0218 & 0.0214 & 0.0219 \\
$k_{\mathrm{r}}^{\theta}(\mathrm{N} \cdot \mathrm{m} / \mathrm{rad})$ & 2814.9 & 2932.4 & 2924.7 & 3356.2 & 2931.0 \\
$\varepsilon_{\mathrm{B}_{1}}(\%)$ & -27.6 & -4.0 & -19.1 & -19.1 & -18.7 \\
$\varepsilon_{\mathrm{B}_{2}}(\%)$ & 4.1 & 0.7 & 3.1 & 3.1 & 3.0 \\
$\varepsilon_{\mathrm{T}_{1}}(\%)$ & 0 & 0 & 0 & 0 & 0 \\
\hline
\end{tabular}

Errors in re-estimating these three frequencies based on the beam equivalent parameters are given in percentages $\left(\varepsilon_{B_{1}, B_{2}, T_{1}}\right)$.

$k_{\mathrm{r}}^{\theta}=2991.8 \pm 187.5 \mathrm{~N} \cdot \mathrm{m} / \mathrm{rad}$. While $\tilde{f}_{\mathrm{B}_{1}}$ is underestimated by about $20 \%$ for all the rackets except $R_{2}$, computations of $\tilde{f}_{B_{2}}$ and $\tilde{f}_{\mathrm{T}_{1}}$ tend to be overestimated by less than $5 \%$.

\subsection{Evolution of player-grip parameters as a function of grip force}

Player-grip equivalent parameters $\left\{k_{\mathrm{h}}, l_{\mathrm{h}}, k_{\mathrm{h}}^{\theta}\right\}$ estimated for the five rackets and the three investigated grip force factors were estimated up to $540 \cdot 10^{3} \mathrm{~N} \cdot \mathrm{m}, 4.4 \cdot 10^{-4} \mathrm{~N} \cdot \mathrm{m}^{2}$ (i.e., a radius $r_{\mathrm{h}}$ up to $6 \mathrm{~cm}$ ), and $1680 \mathrm{~N} \cdot \mathrm{m} / \mathrm{rad}$, respectively. $\left\{k_{\mathrm{h}}, l_{\mathrm{h}}, k_{\mathrm{h}}^{\theta}\right\}$ are related to the grip force index. The greater the grip force, the lower the stiffness $k_{\mathrm{h}}\left(\mathrm{R}_{\mathrm{s}}=-0.53 ; p=0.044\right)$ and the moment of inertia $I_{\mathrm{h}}\left(\mathrm{R}_{\mathrm{s}}=-0.80 ; p<0.01\right)$. On the other hand, the torsional stiffness increased with grip force $\left(R_{s}=\right.$ $0.83 ; p<0.01)$. Estimated values of $\left\{k_{\mathrm{h}}, \mathrm{l}_{\mathrm{h}}, k_{\mathrm{h}}^{\theta}\right\}$ for each racket held in the three assumed grip force conditions are shown in Figure 4. Player-grip parameters follow a similar pattern within the five rackets while no straightforward difference occurred between them. Although the moment of inertia $I_{h}$, as well as the stiffness $k_{\mathrm{h}}$, decreased by $95.8 \pm 4.3 \%$ and $25.2 \pm 2.4 \%$ for a rise of $73.9 \pm 13.1 \%$ in grip force, the torsional stiffness $k_{\mathrm{h}}^{\theta}$ increased by $549.0 \pm 173.7 \%$ for the latter variation in grip force. With $m_{\mathrm{h}}$ fixed to the hand mass of the actual participant, $I_{h}$ variations result in a drop of $85.6 \pm 8.9 \%$ in the equivalent hand radius $r_{\mathrm{h}}$.

Investigating further how variations in player-grip parameters affect the spectral content of rackets, Figure 5 shows the theoretical variations of $\tilde{f}_{\mathrm{B}_{1}}, \tilde{f}_{\mathrm{B}_{2}}$ and $\tilde{f}_{\mathrm{T}_{1}}$ with respect to the linear and torsional parameters $\left\{m_{\mathrm{h}}, k_{\mathrm{h}}\right\}$ and $\left\{I_{\mathrm{h}}, k_{\mathrm{h}}^{\theta}\right\}$, respectively. As expected, while an increase in inertial parameters $\left(m_{\mathrm{h}}\right.$ and $I_{\mathrm{h}}$ ) leads to a frequency decrease, an increase in stiffness parameters $\left(k_{h}\right.$ and $\left.k_{h}^{\theta}\right)$ leads to a frequency increase. Considering then the bending modes, it appears that, depending on the $\left\{m_{\mathrm{h}}, k_{\mathrm{h}}\right\}$ combination, $\tilde{f}_{\mathrm{B}_{1}}$ and $\tilde{f}_{\mathrm{B}_{2}}$ can vary by up to $\pm 50 \%$ and $\pm 20 \%$ respectively around their values under free boundary conditions. Moreover, the results indicate that $k_{\mathrm{h}}$ affects $\tilde{f}_{\mathrm{B}_{1}}$ and $\tilde{f}_{\mathrm{B}_{2}}$ about twice as much as $m_{\mathrm{h}}$. Finally, changes in $\tilde{f}_{\mathrm{T}_{1}}$ are mainly driven by $k_{\mathrm{h}}^{\theta}$ and attain up to 16 times its value under free boundary conditions. Note that, under all conditions, $k_{\mathrm{h}}^{\theta}$ and $l_{\mathrm{h}}$ always lead to an increase in $\tilde{f}_{\mathrm{T}_{1}}$.

\section{Discussion}

This study investigated how the dynamic behaviour of a tennis racket was affected by the grip force and the mechanical characteristics of the racket to obtain a better understanding of the player/racket interaction. This problem has been addressed at both experimental and modelling levels. The first step consisted of gathering the modal responses of five commercial rackets being held with various grip force intensities. In the second step, a phenomenological model of a hand-gripped racket was designed to address the effect of grip force on spectral changes and conversely, in computing the grip force characteristics based on the spectral properties.

Dealing first with the experimental side of the study, the results indicate that the presence of a hand on the racket

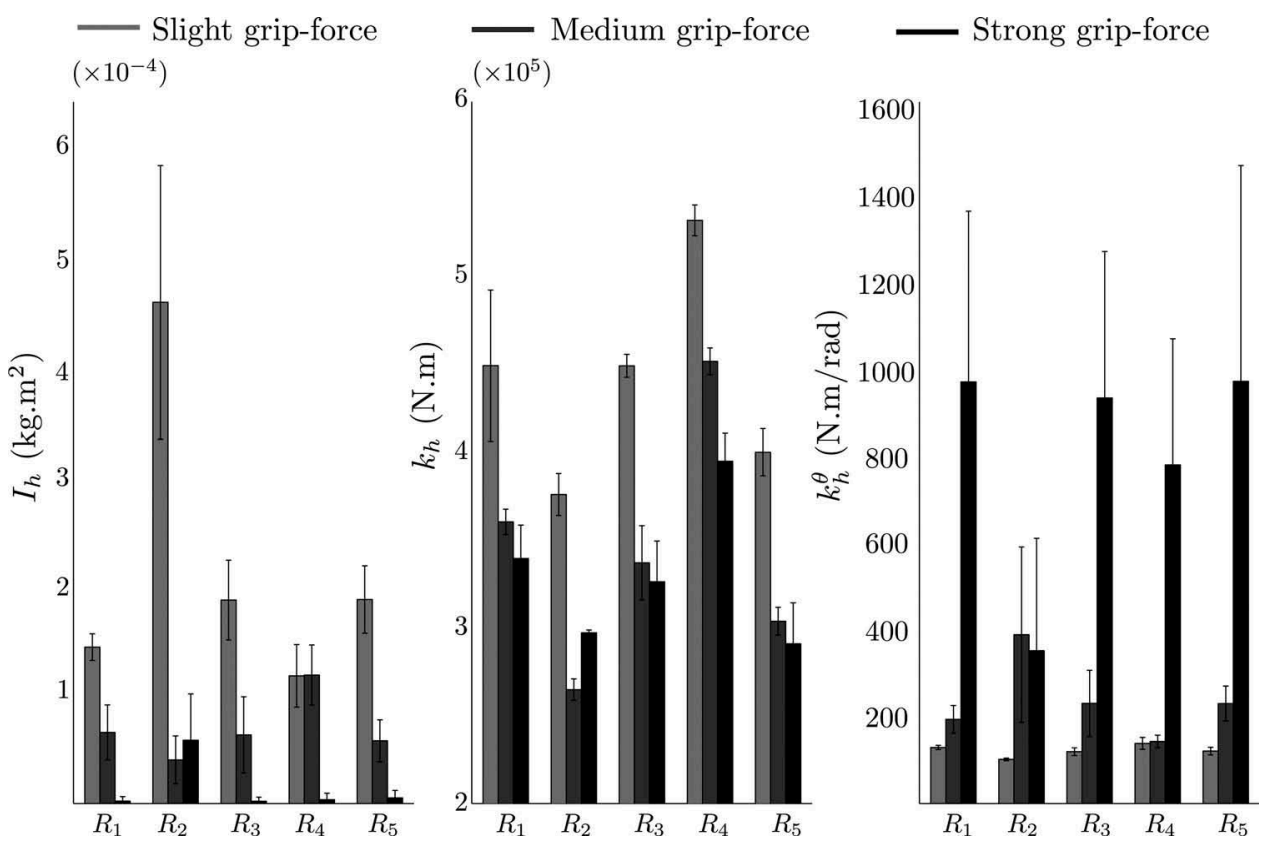

Figure 4. Change in player-grip parameters estimated for the five rackets according to grip force intensity. 

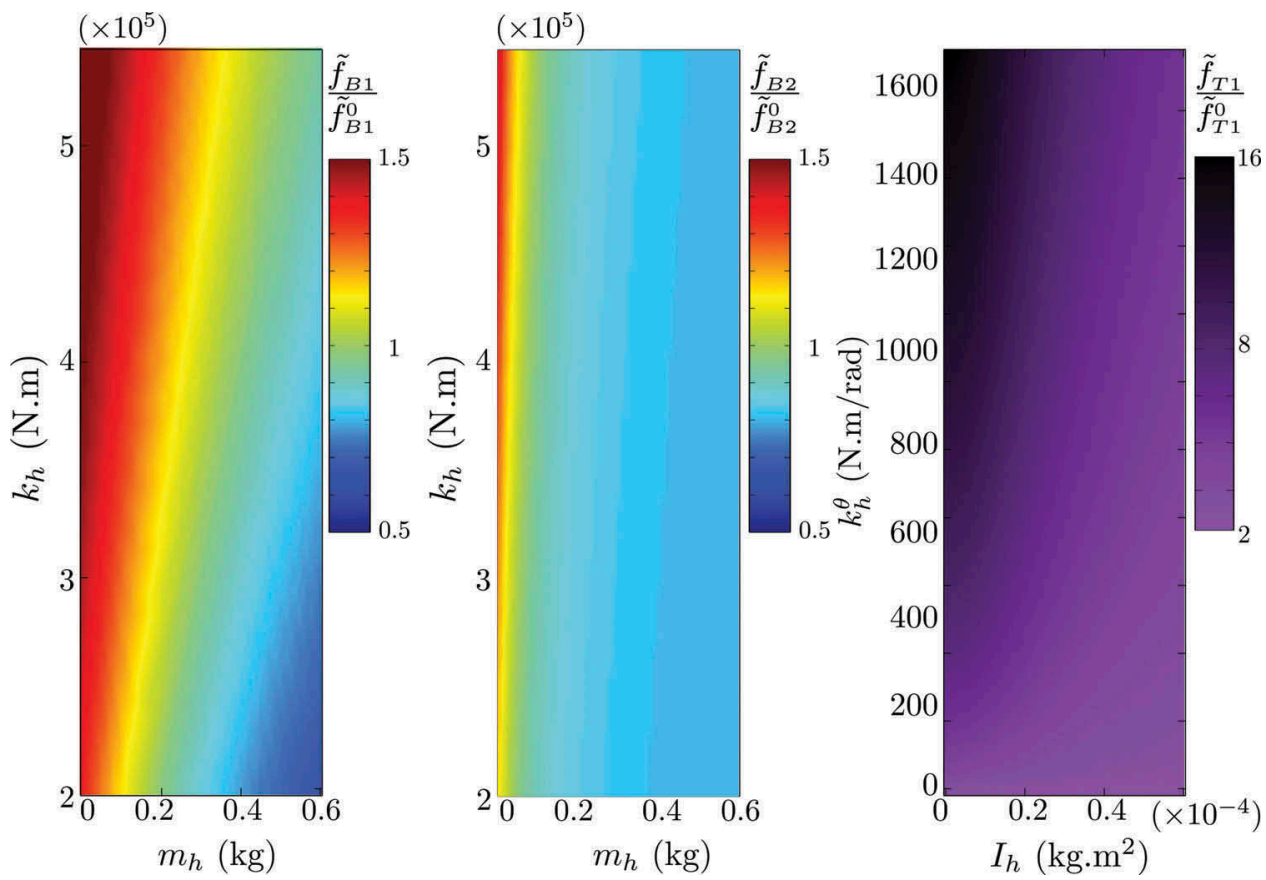

Figure 5. Theoretical evolution of normalised modal frequencies $\left(\tilde{f}_{\mathrm{B}_{1}}, \tilde{f}_{\mathrm{B}_{2}}\right.$ and $\left.\tilde{f}_{\mathrm{T}_{1}}\right)$ according to player-grip parameters. The reference frequencies $\left(\tilde{f}_{\mathrm{B}_{1}}^{0}, \tilde{f}_{\mathrm{B}_{2}}^{0}\right.$ and $\left.\tilde{f}_{\mathrm{T} 1}^{0}\right)$ are determined under free boundary conditions $\left(m_{\mathrm{h}}=k_{\mathrm{h}}=k_{\mathrm{h}}^{\theta}=I_{\mathrm{h}}=0\right)$.

handle greatly affects its dynamic behaviour influencing both modal frequencies and damping factors. Based on modal analysis under free boundary conditions, $f_{\mathrm{B}_{1}}$ and $f_{\mathrm{B}_{2}}$ decrease, $f_{\mathrm{T}_{1}}$ increases and all damping factors greatly increase with grip force. The question of how the hand's presence affects the vibrational behaviour of a piece of equipment has already been addressed by several studies, including sports and musical equipment. For instance, Le Carrou, Chomette, and Paté (2014) have shown that an electric guitarist's body induces a $5 \%$ increase in the damping factor of the first bending mode of his instrument; no straightforward result was obtained for modal frequencies. Moreover, Russell (2012) has stated that the hand provides a great amount of damping on baseball bats and hockey sticks with no significant change in the modal frequencies as compared with the free boundary condition. With regard to the tennis racket, Banwell et al. (2014) outlined spectral modulations between freely suspended and handgripped rackets in three dimensions, with investigations up to $1500 \mathrm{~Hz}$. It has also been indicated that, although the string-bed modes were not affected by the hand, the frequencies of the frame modes decreased relative to the freely suspended case. Our findings are in accordance with these observed phenomena. We observed that the frequency shifts were about $15 \mathrm{~Hz}$ for the first two bending modes (at about 145 and $395 \mathrm{~Hz}$, respectively), and about $6 \mathrm{~Hz}$ for the first torsional mode (at around $370 \mathrm{~Hz}$ ). Such dynamical changes can be expected when an additional element interacts with a structure. However, the frequency and damping factor variations differed from one racket to another because spectral deviations are mostly driven by the location of this element on the structure: no effect occur when a force is applied on a vibrational node of the structure, while significant spectral alterations are expected when the force is located on an anti-node. The player/racket interaction occurs on a vibrational anti-node for the two first bending modes and the first torsional mode, leading to the observed deviation in these modes.

Furthermore, a noteworthy result is that spectral changes (frequency shifts and increases in damping factors) strongly depend on the grip force intensity. Knowing that grip force applied to the handle is adjusted during actual strokes (Knudson, 1991) and according to muscular fatigue (Rossi, 2012), this outcome is likely to indicate that the dynamic behaviour of a racket is modified by the hand grip during actual tennis playing. Given the $500 \mathrm{~Hz}$ upper limit of human skin sensitivity (Goff, 1967) and the [100-320] Hz range of high sensitivity to mechanical vibration, frequencies of the two first bending modes and of the first torsional mode can be perceived. Just-noticeable difference (JND) increases greatly with frequency (Goff, 1967) and is also highly dependent on the excited skin area and the participant's skills (Oey \& Mellert, 2004). We assume hence that variations in the first bending mode frequency due to grip force modulation were perceptible while an accurate estimate of the JND in frequency for tennis players requires further investigation to verify whether or not all other measured spectral changes are perceptible. Moreover, this study has also shown that rackets are not identically affected by grip force, depending on their mechanical characteristics. Assuming that players tune their grip force according the stroke performance (Savage \& Subic, 2006), and to the racket properties (Rossi et al., 2014), this means that, depending on the racket, a tennis player will not modify to the same extent the spectral properties of the stroke-induced vibration propagating into his forearm for a given grip force. Hence, assuming the player is able to perceive these differences, this observation is of great value in the understanding 
of player/racket interaction and its optimisation through grip force control. This assumption is important in considering the player's comfort in racket design.

With regard to the modelling part of the study, we proposed a phenomenological model of the hand-gripped racket in approaching the relationship between grip force and the change in the racket's spectral response. The human hand-arm system has been deeply analysed and modelled investigating its response to vibrating hand-held tools (Rakheja, Wu, Dong, \& Shopper, 2002). However, the key objective of the present model being opposite since we focused on the racket response to the grip force, we decided to develop a simple phenomenological model. For this purpose, we chose to represent the player's effect as a cylindrically shaped massspring system connected to the end of the racket, where the springs reveal both hand and forearm stiffnesses. Obviously, our goal was not to accurately model the racket, since more suitable numerical models have already been proposed (Allen, Hart, Spurr, Haake, \& Goodwill, 2010; Kawazoe \& Yoshinari, 2010), but to design an easily understandable and usable tool for estimating how the modal frequencies of a racket will be affected when held by a player under different grip force conditions. Constraining a racket to a simple three degrees of freedom system, the beam equivalent parameters estimated for each racket are of the expected order of magnitude but not accurately estimated (e.g., about 30\% error for $I_{\mathrm{r}}$ ). Hence, $\tilde{f}_{\mathrm{B}_{1}}$ was poorly described (roughly $20 \%$ error) but $\tilde{f}_{\mathrm{B}_{2}}$ and $\tilde{f}_{\mathrm{T}_{1}}$ were accurately predicted (less than $5 \%$ error). Since the advantage of the model lies in the relative comparison of a given racket's dynamic behaviour as a function of various grip forces as well as in assessing how a racket with a given set of mechanical properties is sensitive to grip force variations, the precision of the racket parameters themselves is not a crucial issue. For a given hand's equivalent mass, a decrease in the linear stiffness induces a decrease in the first bending mode frequency and, to a lesser extent in the second one's frequency. Besides, a decrease in the equivalent hand's radius and $a$ rise in the torsional stiffness both imply a higher first torsional mode.

Several studies have stated that the hand effect on the dynamic behaviour of the racket can be explained by an additional mass (Banwell et al., 2014; Kawazoe \& Yoshinari, 2010). In these studies, the mass of the hand changed with grip force and was significantly lower than the real human hand mass. This outcome can be related to the fall of the apparent mass of the hand under high frequency excitation (from 500 to $100 \mathrm{~g}$ when varying from 100 to $500 \mathrm{~Hz}$ ) (Griffin, 1990). However, the simple addition of a mass on the racket handle is not sufficient to completely represent the player-grip effects we observed on the dynamic behaviour of the racket, especially on the first torsional mode. Although there is no guarantee that changes in this mode affect the tennis player, we assume it is important to take this mode into account since it is directly related to the stroke performance. Indeed, as the handle is an anti-node for the first torsional mode, the handle is likely to have a twisting motion that the hand has to counter in order to stabilise the stroke kinematics. To approximate as closely as possible the anatomical features of the players, we chose to fix the hand mass to that of the actual player, and allowed variations in the cylinder radius as well as in the stiffnesses values of the springs. Results showed that the equivalent hand radius and linear stiffness decrease with grip force while torsional stiffness increases. From a geometrical point of view, as the hand tends to close when increasing the grip force, the equivalent hand radius evolution was expected. Besides, the torsional stiffness goes against the vibrational anti-node of the first torsional mode, and thus favours the hand not to twist with the handle. Therefore, the observed changes in the torsional stiffness were consistent. Eventually, since increasing the grip force produces an increase in muscular force and co-contraction to stabilise the wrist joint, an increase in linear stiffness was also expected. As our observations contradict this assumption, linear stiffness requires further investigation to address its evolution according to grip force. For instance, as proposed by Gurram, Rakheja, Boileau, and Gouw (1996), it could be relevant to introduce non-linear definitions of the model parameters according to grip force.

A limitation of this study is that the modal analyses of the hand-gripped racket are restricted to out-of-plane modes. More accurate operational modal analysis methods would be very advantageous in refining the current measurements and extending them to in-plane modes. This contribution must also be extended to investigate grip force modulation and distribution during actual tennis strokes, and could reveal the propensity of the player to finely control stroke-induced vibration propagating into his forearm during play. Besides, the investigation of the relationships between the racket properties and the evolution of their dynamic behaviour according to grip force would benefit from more rackets, especially with a larger panel of handle sizes or string-bed properties.

\section{Conclusion}

This article has contributed to an understanding of how the dynamic behaviour of a tennis racket changes according to grip force modulations and to the mechanical characteristics of the racket. The most noticeable result is that grip force affects the modal frequencies as well as the damping factors. The stronger the grip force, the higher the damping factors, with lower frequencies for the first two bending modes, and a higher frequency for the first torsional mode. Interestingly, the results indicated further that the dynamic behaviour of a set of rackets was not equally affected by a given grip force. Indeed, their dynamic responses were strongly governed by their mechanical properties. The simple phenomenological model we proposed explains these variations and allows the prediction of the first modal frequencies for a given racket and a given grip force. Overall, this investigation highlights the fact that an item of equipment cannot be designed without taking the human being interacting with it into account. In the particular case of a tennis racket, the grip greatly affects its dynamic behaviour in a frequency range where the human skin is highly sensitive. Whether the grip is voluntarily adjusted or not by the player, this observation is significant with regard to equipment comfort and therefore design. 


\section{Acknowledgements}

The authors would like to thank the Decathlon company for the loan of the rackets involved in this study, and the referees for their valuable comments which helped to improve the manuscript.

\section{Disclosure statement}

No potential conflict of interest was reported by the authors.

\section{References}

Allen, T., Hart, J., Spurr, J., Haake, S., \& Goodwill, S. (2010). Validated dynamic analysis of real sports equipment using finite element; a case study using tennis rackets. Proceedings of the 8th Conference of the International Sports Engineering Association (ISEA). Vienna, Austria, pp. 3275-3280.

Banwell, G. H., Roberts, J. R., Halkon, B. J., Rothberg, S. J., \& Mohr, S. (2014). Understanding the dynamic behaviour of a tennis racket under play conditions. Experimental Mechanics, 54, 527-537. doi:10.1007/s11340013-9803-9

Brody, H. (1979). Physics of the tennis racket. American Journal of Physics, 47(6), 482-487. doi:10.1119/1.11787

Brody, H. (1987). Models of tennis racket impacts. International Journal of Sport Biomechanics, 3, 293-296.

Brody, H. (1989). Vibration damping of tennis rackets. International Journal of Sport Biomechanics, 5, 451-456.

Cross, R. (1997). The dead spot of a tennis racket. American Journal of Physics, 65(8), 754-764. doi:10.1119/1.18646

Cross, R. (2000). Flexible beam analysis of the effects of string tension and frame stiffness on racket performance. Sports Engineering, 3, 111-122. doi:10.1046/j.1460-2687.2000.00046.x

Cross, R., \& Nathan, A. (2009). Performance versus momentof-inertia of sporting implements. Sports Technology, 2(1-2), 7-15. doi:10.1080/ 19346182.2009.9648493

Goff, G. D. (1967). Differential discrimination of frequency of cutaneous mechanical vibration. Journal of Experimental Psychology, 74, 294-299. doi:10.1037/h0024561

Griffin, M. J. (1990). Handbook of human vibration. London: Academic Press Edition. ISBN 0123030412.

Guillaume, P., Verboven, P., Vanlanduit, S., Van Der Auweraer, H., \& Peeters, B. (2003). A poly-reference implementation of the least-squares complex frequency-domain estimator. Proceedings of the IMAC XXI, Kissimmee, FL.

Gurram, R., Rakheja, S., Boileau, P. E., \& Gouw, G. J. (1996). Development of a grip force dependent hand-arm vibration model. Central European Journal of Public Health, 4(1), 65-73.

Hatze, H. (1976). Forces and duration of impact, and grip tightness during the tennis stroke. Medicine \& Science in Sports \& Exercise, 8, 88-95. doi:10.1249/00005768-197600820-00014

Hennig, E. M. (2007). Influence of racket properties on injuries and performance in tennis. Exercise and Sport Sciences Reviews, 35(2), 62-66.

Hennig, E. M., Rosenbaum, D., \& Milani, T. L. (1992). Transfer of tennis racket vibrations onto the human forearm. Medicine \& Science in Sports \& Exercise, 24(10), 1134-1140. doi:10.1249/00005768-199210000-00011

Kawazoe, Y., \& Yoshinari, K. (2010). Prediction of the impact shock vibrations of the tennis player's wrist joint: Comparison between two super large sized tennis rackets with different frame weight distributions. Journal of System Design and Dynamics, 4(2), 331-347. doi:10.1299/jsdd.4.331

Knudson, D. V. (1991). Forces on the hand in the tennis one-handed backhand. International Journal of Sport Biomechanics, 7, 282-292.

Kotze, J., Mitchell, S. R., \& Rothberg, S. J. (2000). The role of the racket in high-speed tennis serves. Sports Engineering, 3, 67-84. doi:10.1046/ j.1460-2687.2000.00050.x

Le Carrou, J.-L., Chomette, B., \& Paté, A. (2014). Influence of the instrumentalist on the electric guitar vibratory behaviour. Proceedings of the 21th International Symposium on Musical Acoustics (ISMA 2014), Le Mans, France, pp. 413-417.
Le Carrou, J.-L., Gautier, F., \& Badeau, R. (2009). Sympathetic string modes in the concert harp. Acta Acustica United with Acustica, 95, 744-752. doi:10.3813/AAA.918202

Miller, S. (2006). Modern tennis rackets, balls and surfaces. British Journal of Sports Medicine, 40(5), 401-405. doi:10.1136/bjsm.2005.023283

Oey, H., \& Mellert, V. (2004). Vibration thresholds and equal vibration levels at the human fingertip and palm. Proceedings of the 18th International Congresses on Acoustics, Kyoto, Japan, pp. 1-4.

Paté, A., Le Carrou, J.-L., \& Fabre, B. (2014). Predicting the decay time of solid body electric guitar tones. The Journal of the Acoustical Society of America, 135(5), 3045-3055. doi:10.1121/1.4871360

Piranda, J. (2001). Analyse modale expérimentale [Experimental modal analysis]. Techniques de l'ingénieur - Mesures acoustiques et vibratoires. TIB420DUO(R6180).

Rakheja, S., Wu, J. Z., Dong, R. G., \& Shopper, A. W. (2002). A comparison of biodynamic models of the human hand-arm system for applications to hand-held power tools. Journal of Sound and Vibration, 249(1), 55-82. doi:10.1006/jsvi.2001.3831

Rossi, J. (2012). Analyse biomécanique de l'interface main-raquette lors de la pratique du tennis: Applications à l'étude du tennis elbow (Dissertation). University of Aix-Marseille, p. 169.

Rossi, J., Berton, E., Grélot, L., Barla, C., \& Vigouroux, L. (2012). Characterisation of forces exerted by the entire hand during the power grip: Effect of the handle diameter. Ergonomics, 55, 682-692. doi:10.1080/00140139.2011.652195

Rossi, J., Vigouroux, L., Barla, C., \& Berton, E. (2014). Potential effects of racket grip size on lateral epicondilalgy risks. Scandinavian Journal of Medicine \& Science in Sports, 24, e462-e470. doi:10.1111/sms.2014.24.issue-6

Roy, R., Paulraj, A., \& Kailath, T. (1986). ESPRIT - A subspace rotation approach to estimation of parameters of cisoids in noise. IEEE Transactions on Acoustics, Speech and Signal Processing, 34, 13401342. doi:10.1109/TASSP.1986.1164935

Russell, D. A. (2012). Flexural vibration and the perception of sting in handheld sports implements. Proceedings of the 41st International Congress and Exposition on Noise Control Engineering 2012 (INTER-NOISE 2012), New York, NY.

Savage, N. (2006). Vibration absorption in the tennis grip and the effects on racquet dynamics (Unpublished doctoral dissertation). School of Aerospace, Mechanical and Manufacturing Engineering, RMIT University, Melbourne, Australia.

Savage, N., \& Subic, A. (2006). Relating grip characteristics to the dynamic response of tennis racquets. The Engineering of Sport, 6, 155-160.

Stroede, C. L., Noble, L., \& Walker, H. S. (1999). The effect of tennis racket string vibration dampers on racket handle vibrations and discomfort following impacts. Journal of Sports Sciences, 17, 379-385. doi:10.1080/ 026404199365894

Wei, S.-H., Chiang, J.-Y., Shiang, T.-Y., \& Chang, H.-Y.. (2006). Comparison of shock transmission and forearm electromyography between experienced and recreational tennis players during backhand strokes. Clinical Journal of Sport Medicine, 16(2), 129-135. doi:10.1097/00042752-200603000-00008

Zatsiorsky, V. (2002). Kinetics of human motion (p. 672, pp. 594). Champaign, IL: Human Kinetics Publishers.

\section{Appendix}

\section{Computation of player-grip parameters}

Assuming the \{uniform beam; mass-springs\} system to be conservative and described by generalised coordinates $\{\Phi\}$, the Euler-Lagrange equations describe its dynamic behaviour in a free oscillating regime by

$$
\frac{\mathrm{d}}{\mathrm{d} t}\left[\frac{\partial \mathcal{L}}{\partial \dot{\Phi}_{i}}\right]-\frac{\partial \mathcal{L}}{\partial \Phi_{\mathrm{i}}}=\{0\}
$$

where $\mathcal{L}$ is the Lagrangian function defined by the difference between the system's kinetic and potential energies, $\mathcal{T}$ and $\mathcal{V}$, respectively. $\mathcal{T}$ and $\mathcal{V}$ are expressed as

$$
\mathcal{T}=\frac{1}{2}\{\dot{\Phi}\}^{\top} \mathbb{M}\{\dot{\Phi}\}
$$


and

$$
\mathcal{V}=\frac{1}{2}\{\Phi\}^{\top} \mathbb{K}\{\Phi\}
$$

$\{\Phi\}$ are the generalised coordinates of the system, and $\mathbb{M}$ and $\mathbb{K}$ are square matrices containing the masses and bending and torsional stiffnesses of the system, namely

$$
\mathbb{M}=\left[\begin{array}{cc}
\frac{m_{\mathrm{r}}}{2} \mathbb{I}_{N_{\mathrm{b}}}+m_{h}(-1)^{i+j} & \mathbb{O}_{N_{\mathrm{b}}, N_{\mathrm{t}}} \\
\mathbb{O}_{N_{\mathrm{t}}, N_{\mathrm{b}}} & \frac{l_{\mathrm{r}}}{2} \mathbb{I}_{N_{t}}+I_{\mathrm{h}}(-1)^{i+j}
\end{array}\right],
$$

and

$$
\mathbb{K}=\left[\begin{array}{cc}
\mathbb{K}_{1} & \mathbb{O}_{N_{\mathrm{b}}, N_{\mathrm{t}}} \\
\mathbb{O}_{N_{\mathrm{t}}, N_{\mathrm{b}}} & \mathbb{K}_{2}
\end{array}\right],
$$

where $\mathbb{K}_{1}=\operatorname{diag}\left\{\frac{8 \pi^{4}}{2} k_{\mathrm{r}}, \ldots, \frac{\left(N_{\mathrm{b}}+1\right)^{4} \pi^{4}}{2} k_{\mathrm{r}}\right\}+k_{\mathrm{h}}(-1)^{i+j}$,

$\mathbb{K}_{2}=\operatorname{diag}\left\{\frac{4 \pi^{2}}{2} k_{\mathrm{r}}^{\theta}, \ldots, \frac{\left(N_{t}+1\right)^{2} \pi^{2}}{2} k_{\mathrm{r}}\right\}+k_{\mathrm{h}}^{\theta}(-1)^{i+j}, i$ and $j$ are indices specifying the matrices' rows and columns, and $N_{\mathrm{b}}$ and $N_{\mathrm{t}}$ are the numbers of degrees of freedom in bending and torsion, respectively. Equation (6) therefore gives $N_{\mathrm{b}}+N_{\mathrm{t}}$ uncoupled equations

$$
\mathbb{M}\{\ddot{\Phi}\}+\mathbb{K}\{\Phi\}=\{0\}
$$

As the generalised coordinates $\{\Phi\}$ are harmonics in angular frequencies $\omega$, Equation (11) leads to

$$
\left(\mathbb{K}-\mathbb{M} \omega^{2}\right)\{\Phi\}=\{0\},
$$

where the angular frequencies $\omega$ can be determined by solving

$$
\left|\mathbb{K}-\mathbb{M} \omega^{2}\right|=\{0\} .
$$

